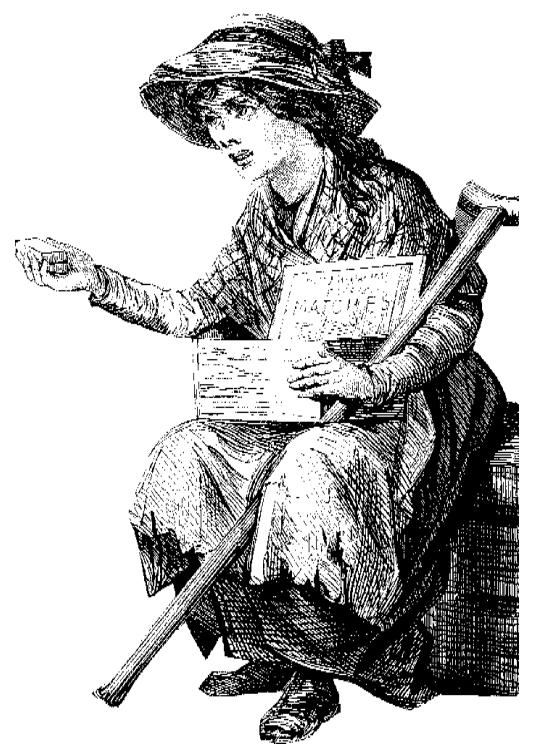

\title{
LAS JORNADAS DE RECUPERACIÓN: NEGACIÓN A LOS DIFERENTES RITMOS DE APRENDIZAJE'
}

\author{
Édgar Tamayo Sánchez \\ Profesor Instituto Ecológico El Carmen \\ Catedrático Universidad de La Guajira \\ Sudy Gutiérrez Chinchía \\ Miriam Morales Bolívar \\ Profesoras Colegio Livio Reginaldo Fischioni
}

Es hoy un elemento común en los colegios de educación formal, la creación de espacios académicos, para abordar la problemática que tiene que ver con las dificultades que presentan los estudiantes durante el desarro-

\footnotetext{
${ }^{1}$ Este documento hace parte de un trabajo investigativo que se desarrolla actualmente el colegio Livio Reginaldo Fischioni de Riohacha, en el marco de la Especialización en Evaluación Escolar de la Universidad de La Guajira.
}

llo de los procesos educativos en las diversas áreas o asignaturas. En algunas instituciones, la creación de estos espacios obedece a la necesidad de cumplir con el mandato de la norma (Decreto 1860/94), en otras, la creación de estos espacios es consecuencia de la problematización que se hace a propósito de la evaluación; podríamos decir que son niveles de elaboración alcanzados dentro de un proceso de reflexión, motivado por el ejerci- cio de las prácticas evaluativas y por los resultados obtenidos mientras se realizan estas prácticas. Sin embargo, en ambos casos se trata de establecer momentos dentro del calendario académico para reunir a los estudiantes de un mismo grado que no pudieron "alcanzar logros" establecidos en los programas de estudio. Para determinar cuáles son estos estudiantes se toman como parámetros aquellos que sí alcanzaron los logros. Este 
otro grupo de estudiantes permite establecer, por comparación, quienes tienen dificultades en su proceso de aprendizaje, pero además permite justificar que sí se hizo un "buen" trabajo pedagógico por parte del profesor.

En el marco del Decreto 1860/ 94 podemos encontrar las disposiciones que obligan a las instituciones educativa a la creación de los espacios académicos, al cual deben asistir aquellos estudiantes que necesitan "superar fallas o limitaciones en la consecución de los logros", pero también, encontramos un discurso que evidencia la racionalidad desde la cual se hacen dichas exigencias.

El articulo 52 de dicho decreto establece que: "La promoción en la educación básica se fundamenta en el reconocimiento de la existencia de diferencias en el ritmo de aprendizaje de los alumnos. Por tanto, los educandos deben tener oportunidades de avanzar en el proceso educativo, según sus capacidades y aptitudes personales".

Sin embargo, dice ésta misma norma en su articulo 49, "después de la evaluación de cada periodo, el docente programará como parte de las labores normales del curso, las actividades grupales o individuales que requieran para superar fallas o limitaciones en la consecución de los logros por parte de los alumnos". Esto significa que no hay tal reconocimiento a los diferentes ritmos de aprendizaje, puesto que se establece un momento limite (final del periodo) durante el proceso educativo para que todos los estudiante hayan al- canzado los logros propuestos previamente por el profesor. Es decir; se promueve con la práctica evaluativa la homogeneidad o uniformidad del estado intelectual de los estudiantes, lo cual indica que hay un reconocimientos expreso, por parte de la norma (Decreto 1860/94), en que si en un momento determinado algunos estudiantes no han alcanzado los logros, es porque tienen dificultades o algún tipo de limitaciones en su capacidad para aprender y no porque tienen diferentes ritmos de aprendizaje, por lo tanto, se le deben programar "actividades grupales o individuales que requieran para superar fallas o limitaciones".

Vemos que a pesar de que se habla de un reconocimiento a los diferentes ritmos de aprendizaje, subyace la idea de la carencia de una capacidad potencial para aprender. Se ven en consecuencia, dos tendencias opuestas desde las cuales hay que asumir la práctica evaluativa: una que reclama la mirada desde la diferencia en los ritmos de aprendizaje y la otra que propende por la homogeneidad y la estandarización en el aprendizaje de los estudiantes. Esta última es la que se impone y, a partir de ésta idea, es que se asume el acto evaluativo. Desde éste presupuesto es que se han creado los "cursos remediales", "cursos de inducción", "cursos nivelatorios", entre otros.

Esta es la creencia que existe en un número considerable de nuestras instituciones educativas, es así como hoy aparecen en las escuelas dichas actividades con los nombres de: "Actividades de superación", "Jornadas de recu- peración", "Actividades pedagógicas complementarias", etc.

Con estas actividades se pretende "nivelar" al estudiante para que vuelva al aula de clases en condiciones de igualdad con respecto a los demás (Cf. Jurado, 1998). Se trata de que todos deben estar con la misma cantidad de logros alcanzados, como condición necesaria para que haya "normalidad académica" en el desarrollo de los programas de estudio.

En el marco de éstas ideas, son significativos los pronunciamientos de algunos profesores cuando se les preguntó sobre el sentido que tenía para ellos las jornadas de recuperación. Al respecto expresaron ${ }^{2}$ :

- Es una oportunidad que se le brinda al estudiante para superar los vacíos que quedaron en determinado tema.

- Que el estudiante alcance los logros que no pudieron obtener durante el periodo.

- Las jornadas de recuperación se realizan con el fin de que el estudiante supere las insuficiencias que ha tenido en los temas que se han estudiado en cada uno de los periodos del año lectivo.

Existe el convencimiento por parte del profesor en que si el estudiante no alcanza todos los logros propuestos es porque tiene dificultades en su capacidad de aprendizaje, en consecuencia, son remitidos a las jornadas de recuperación cuando presen-

\footnotetext{
${ }^{2}$ En adelante lo escrito en bastardilla corresponde a las voces de maestros y estudiantes con los cuales interactuamos.
} 


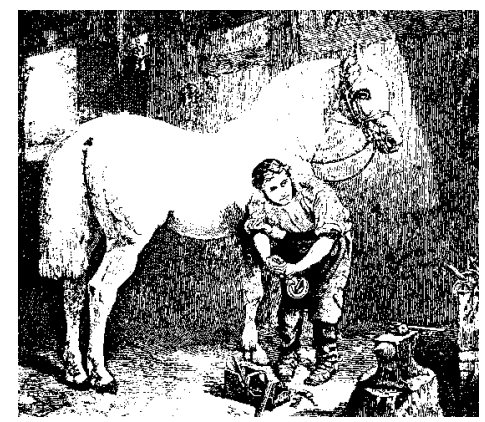

ta insuficiencias en el manejo de algunos temas, o como argumenta otro maestro, cuando presenta deficiencias en la asimilación de logros establecidos.

Debido a este convencimiento, las actuaciones del maestro tienden a que los estudiantes "superen" tales dificultades o "deficiencias", por tal razón durante las jornadas de recuperación sólo se evalúa el conocimiento sobre donde se presenta la insuficiencia ó también, conceptos y temas en los cuales no se han alcanzado los logros en su totalidad.

Estos imaginarios no dejan mirar otras cosas del ámbito evaluativo, como por ejemplo, ¿Por qué es el estudiante quien debe someterse a las jornadas de recuperación? ¿El acto pedagógico, los programas de estudio, los roles de los sujetos dentro del aula escolar, incluso las mismas prácticas evaluativas, merecen remitirse a dichas jornadas? o ir un poco más allá y cuestionarse sobre la validez de la hipótesis del déficit en la capacidad de aprendizaje de los estudiantes y, en consecuencia, indagar por la pertinencia de las jornadas de recuperación que, en la actualidad, se están llevando a cabo en el colegio.
Los estudiantes, por su parte, no son ajenos a estas concepciones. En sus imaginarios persiste también la idea de tener que ser remitidos a las actividades escolares que les permitan "superar las deficiencias" que según sus profesores han tenido durante el periodo académico. No son pocas las afirmaciones que se manifiestan en tal sentido: Las recuperaciones yo pienso que no siempre debemos hacerlas igual, puesto que nos hacen previas y uno las pierde. Deberían como cambiar la metodología como se toman las notas, siempre se hacen evaluaciones este estudiante a pesar de estar en desacuerdo con la forma como se realiza la evaluación en las jornadas de recuperación, no pone en cuestión el hecho de tener que "recuperar". En sus expresiones se pue- de observar que de cierta manera se validan dichas prácticas.

\section{Análisis de algunos instrumentos}

Como es un mandato de la normatividad, las escuelas se han visto obligadas a programar periódicamente (al finalizar cada periodo) ${ }^{3}$ las llamadas "jornadas de recuperación" ó "actividades de superación”. Así por ejemplo, en el Colegio Livio Reginaldo Fischioni de Riohacha se tomó una semana aproximadamente para citar a los estudiantes que debían "recuperar" en algún área del conocimiento, en aquellos temas en los que, hasta la fecha, habían tenido problemas académicos. A continuación mostramos el calendario con el cual se planearon dichas actividades (sic):

\begin{tabular}{|l|l|l|c|}
\hline \multicolumn{4}{|c|}{ RECUPERACIÓN DEL PRIMER PERIODO } \\
\hline & ÁREAS & HORA & FECHA \\
\hline 1 & Inglés & $6: 30-7: 15$ a.m. & 27 julio \\
2 & Sociales & $7: 15-8: 00$ a.m. & \\
\hline 1 & Castellano - Filosofía & $6: 30-7: 15$ a.m. & 28 julio \\
2 & Geometría & $7: 15-8: 00$ a.m. & \\
\hline 1 & Matemáticas & $6: 30-7: 15$ a.m. & 29 julio \\
2 & Ética y Valores humanos & $7: 15-8: 00$ a.m. & \\
\hline 1 & Biología - Química & $6: 30-7: 15$ a.m. & 30 julio \\
2 & Ecología & $7: 15-8: 00$ a.m. & \\
\hline 1 & Religión - Democracia & $6: 30-7: 15$ a.m. & 31 julio \\
2 & Educación artística & $7: 15-8: 00$ a.m. & \\
\hline 1 & Educación física & $6: 30-7: 15$ a.m. & 3 agosto \\
\hline
\end{tabular}

\footnotetext{
${ }^{3}$ En el colegio Livio Reginaldo Fischioni el año lectivo se divide en tres periodos académicos, el calendario que aquí mostramos corresponde al primero de ellos, cuya duración iba desde el 2 de febrero hasta el 1 de mayo.
}

El cuadro anterior apareció en cartelera, y nos da una idea de la noción que sobre "jornadas de recuperación" circula en esta institución. 
A cada área se le asigna un día dentro de éste calendario y durante éste día, se estipula un tiempo de 45 minutos para realizar las actividades programadas. Dicho de otra forma, los estudiantes tienen 45 minutos para "recuperar" los logros del periodo académico en el cual han tenido dificultades y el maestro para "nivelar" a estos estudiantes con el resto del grupo; es decir, para lograr obtener un grupo académicamente homogéneo.

Existen otros instrumentos que pueden aportarnos elementos para ir desentrañando el sentido de la evaluación que se realiza durante las "jornadas de recuperación". A continuación nos ocuparemos de dos pruebas escritas que, por considerarlas relevantes, fueron seleccionadas de un grupo de exámenes de diferentes áreas, realizados por estudiantes de diversos grados.

La primera prueba (ver examen 1), corresponde a la asignatura de Ecología, en la cual el maestro formuló el siguiente cuestionario:

1. Explique por qué se deben proteger las zonas de nacimiento de agua.

2. ¿Por qué debemos proteger la biodiversidad?

3. ¿Por qué fue creado el Ministerio del medio ambiente?

Las preguntas tienden a buscar la reflexión del estudiante en torno al problema ecológico, específicamente sobre la conservación del Medio Ambiente. Son preguntas que abren espacios para que el estudiante manifieste sus ideas sobre el tema que, supuestamente, ha sido motivo de discusión durante algunas clases, pero en el cual, el alumno no pudo alcanzar los logros trazados por el profesor.

Con este cuestionario, elaborado por el profesor teniendo en cuenta los temas en los cuales el joven ha tenido dificultades de tipo académico, se pretende promover su "recuperación". Es decir, se quiere que con esta reflexión el estudiante supere las dificultades presentadas en ésta área del conocimiento. Sin embargo, aparentemente, las respuestas dadas no convocan la interlocución del profesor, puesto que él no explicita expresiones que conduzcan a establecer el diálogo con el escrito del estudiante, sólo se limita a dar una calificación.

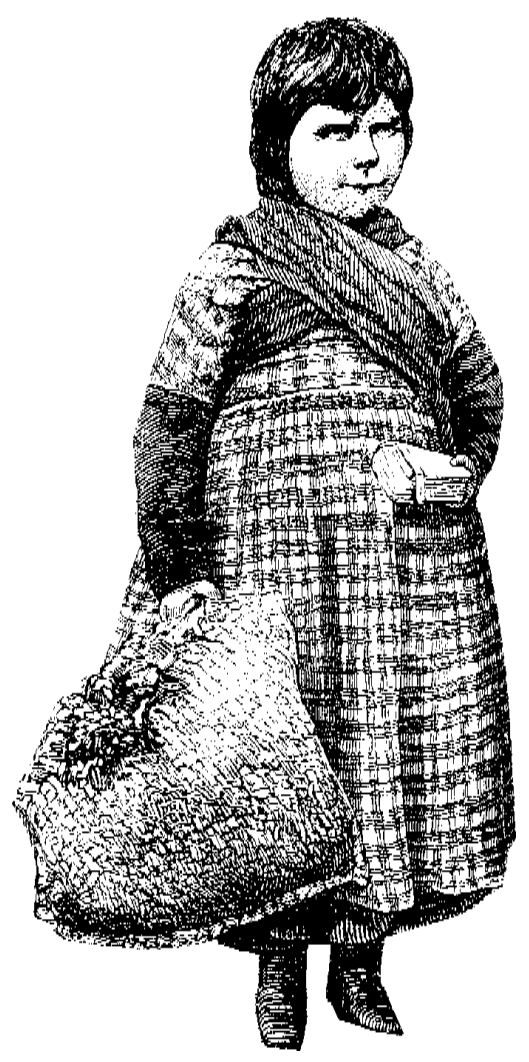

Aunque las respuestas a las preguntas formuladas podrían informar sobre el proceso de construcción de conocimiento al respecto del tema, sobre los niveles de problematización y, por supuesto, sobre el interés que el estudiante manifiesta en torno a la temática, la intención del maestro no está focalizada hacia este tipo de indagaciones, la evaluación se queda entonces en una mirada muy superficial que en la expresión escrita sólo es capaz de decir que el estudiante merece una "B".

La segunda prueba escrita (ver examen 2), corresponde al área de Español, y fue propuesta a varios estudiantes del grado noveno $\left(9^{\circ}\right)$ durante las "Jornadas de Recuperación”. En ella el profesor planteó los siguientes interrogantes:

1. En cuántas partes se divide la oración subordinada y dé ejemplos.

2. En cuántas partes se divide la oración coordinada y dé ejemplos.

3. ¿Qué es oración?

4. ¿Qué es oración simple y oración compuesta? Dé ejemplos.

Se pide aquí, mediante este cuestionario, que el estudiante recuerde algunas definiciones y luego las aplique en la elaboración de ejemplos. Lo relevante entonces, ya que es lo que promueve este tipo de exámenes, es memorizar las definiciones, por lo tanto la evaluación que se haga estará orientada a mirar la capacidad de memorización de los estudiantes. No importa mucho la competencia para analizar textos, ni la producción de escritos 


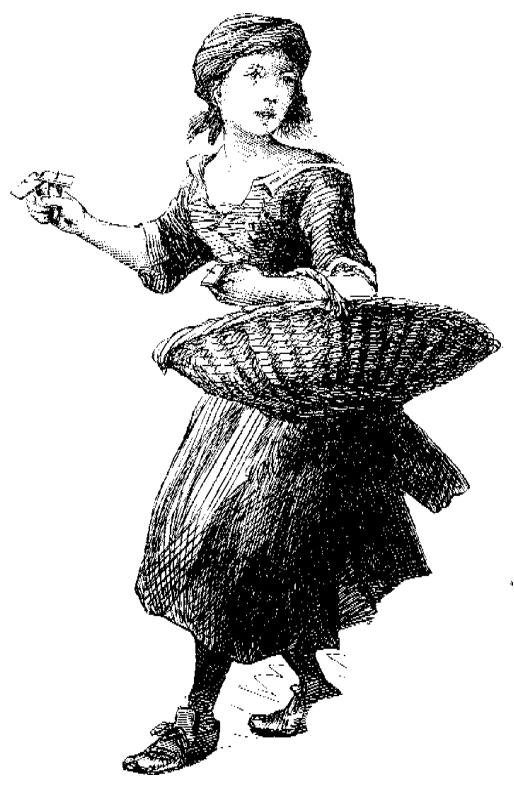

que puedan surgir de dichos análisis o de la vivencia que cada uno tiene durante su permanencia en el aula de clases y, en general, en la institución educativa.

Estos ejemplos son apenas una muestra de lo que sucede con la evaluación durante las llamadas "Jornadas de Recuperación" (o actividades de superación según los artículos 49 y 50 del Decreto 1860/94). Aunque los ejemplos son tomados en las áreas de español y ecología, la situación no es ajena a las demás áreas del conocimiento.

Lo anterior implica que la evaluación que se realiza durante las "Jornadas de Recuperación" no se diferencia en nada con la evaluación que se realiza durante los diversos momentos y eventos que a diario se viven en el proceso educativo. Esto lo podemos constatar en la afirmación que hizo un estudiante durante una entrevista: El profesor debe cambiar este método porque si siempre va a hacer previa y ve que el alumno tiene deficiencias en la previa pero el alumno puede rendir de otra forma. A pesar de que se habla de una evaluación integral, cualitativa, observamos que las prácticas evaluativas desarrolladas durante las "Jornadas de Recuperación" se reducen a la presentación de cuestionarios por parte del maestro y a la solución de ellos por parte de los estudiantes. Al respecto un estudiante señala que: creo que es mejor que nos sigan explicando el tema que perdimos y después nos hacen la evaluación.

Con los resultados obtenidos se clasifican a los estudiantes en una escala ascendente que va desde "I" (insuficiente), pasa por un nivel intermedio "B" (bueno) y termina con un nivel máximo "E" (excelente). Esta forma de asignar una nota al alumno es considerado por el docente como "confiable", es el reflejo numérico de lo que el alumno sabe. Como dice Virginia Aguilar (Pérez y Bustamante, 1996: 91) "La cuantificación permite legitimar el ejercicio de la autoridad sobre el alumno, asignando a este un número que lo clasifica como superior o inferior a otros, desconociéndose a su vez una serie de elementos tanto interno (procesos afectivos cognitivos) como externos (ambiente sociocultural) que han influido directamente en el proceso de aprendizaje".

\section{En la búsqueda de una posible alternativa}

De toda ésta situación planteada, surgen interrogantes que merecen detenerse un poco para hacer una mirada exhaustiva que busque explicaciones y sentidos para empezar a tomar distancias de las prácticas pedagógicas y de las concepciones que, sobre evaluación, circulan en nuestra institución. Algunos interrogantes son: ¿Qué elementos de nuestro tejido social hacen que las prácticas evaluativas que se realizan, existan en nuestro contexto y tiendan a perpetuarse sin sufrir modificaciones significativas? ¿En qué contribuyen nuestras acciones para que la situación problémica denunciada exista? y en tal sentido ¿Cómo podríamos abordar el acto pedagógico para generar relaciones que propendan por la construcción colectiva del conocimiento y por la construcción de una realidad que atienda nuestra cultura y nuestra historia?

Es obvio que debemos asumir unos presupuestos teóricos diferentes a los que hablan de un déficit en la capacidad de aprendizaje de los estudiantes, "se trata más bien de la identificación de regulaciones culturales y de modelos pedagógicos, como determinantes en los diferentes ritmos de aprendizaje, no sólo de los niños sino de todas las personas involucradas en el sistema educativo" (Jurado, 1998: 27). Creemos que por ser diferentes en nuestros pensamientos y por tener diferentes niveles de compromiso frente al evento educativo, tenemos también diferentes ritmos de aprendizaje. Es desde aquí que proponemos asumir el acto evaluativo, para iniciar una ruptura con las ideas que buscan la homogeneización y la estandarización de los sujetos y, en lugar de ello, hacer un reconocimiento a las 


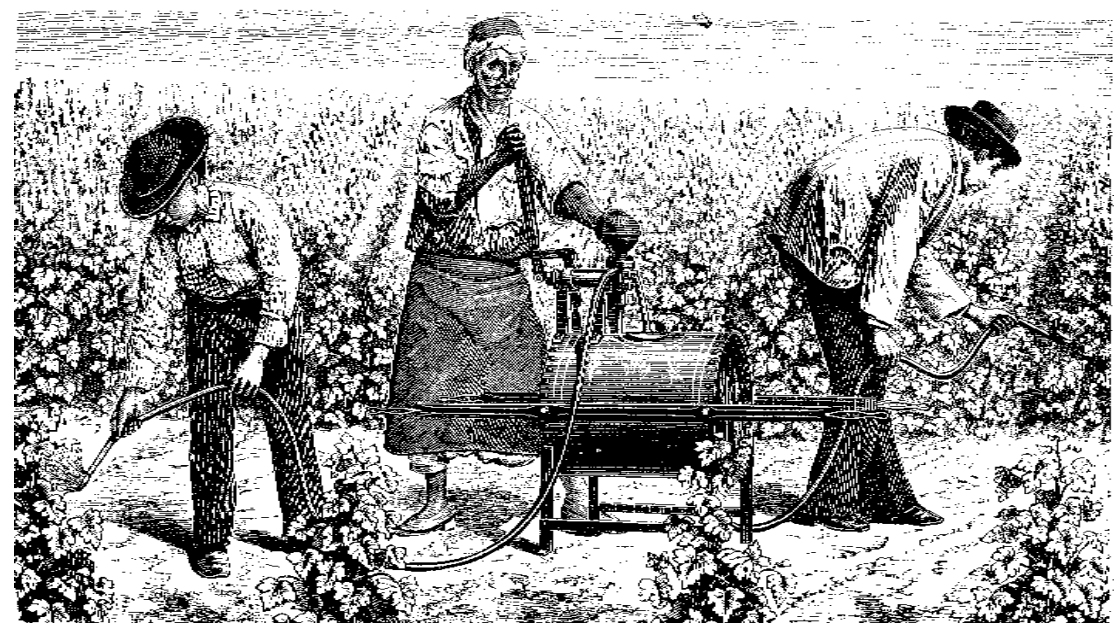

individualidades, a los diferentes niveles de compromiso con la formación y la estructuración intelectual, a los diferentes niveles de elaboración conceptual, etc.

Pero asumir estos presupuestos requiere repensar la escuela, o sea, empezar a cuestionar lo que Lilian Caicedo (1998) denomina los "consolidados"; dicho de otra forma, empezar a sospechar de ciertos elementos que constituyen la realidad escolar en que vivimos, elementos que han sido aceptados como verdades, con los cuales, hemos vivido, y que por lo tanto, han adquirido el carácter de inobjetables. Requiere en consecuencia, asumir la práctica pedagógica desde una actitud investigativa, desde una interacción constante con el otro o con los otros.

Consideramos que es desde la experiencia investigativa que se puede hacer evaluación cualitativa, en donde la etnografía juega un papel definitivo, puesto que se trata de dar cuenta del acompañamiento a los estudiantes en el aula durante los dife- rentes procesos de formación; esto es, se trata de indagar por "las trayectorias a través de las cuales los sujetos se producen cognitiva, afectiva y socioculturalmente" (Jurado y otros, 1998: 24).

Las anteriores ideas nos permiten ir configurando algunos criterios evaluativos que procuran tomar distancia de la tecnología educativa, de la medición, de la evaluación por logros y por objetivos, de la estandarización y homogeneización de los grupos, de la promoción y reprobación, del éxito o el fracaso, etc. Dichos criterios deberán atender aspectos como los siguientes:

- La heterogeneidad del grupo es un elemento que enriquece los procesos de formación, por cuanto es desde la diversidad de ideas y de conceptos como pueden generarse los debates académicos que propician la construcción social de conocimientos.

- La evaluación es un momento del acto pedagógico que da cuenta del acompaña- miento que se le hace al estudiante durante el proceso de construcción de conocimientos.

- La evaluación promueve la formación intelectual de los sujetos, por tanto promueve también toda interlocución (partimos de la consideración de que el conocimiento es una construcción social), en especial con la comunidad educativa en la cual se esta inmerso.

- Las respuestas que dan los estudiantes a las pruebas formuladas por sus maestros, evidencian los niveles alcanzados en la construcción de conocimientos en torno de una temática específica y dichas respuestas, no necesariamente deben tener los mismos niveles de elaboración. En este sentido, las pruebas deben estar focalizadas, no a determinar cuál es la posición de un estudiante con respecto a los demás del grupo como comúnmente se viene haciendo, sino a establecer su posición frente a la temática objeto de estudio y hacia la búsqueda de situaciones problémicas que le dificultan a los estudiantes la construcción de conocimientos y/o hacia la búsqueda de los obstáculos epistemológicos que le impiden al maestro desarrollar competencias pedagógicas y didácticas en su ejercicio de la enseñanza.

- Hacer evaluación cualitativa exige asumir el acto pedagógico desde una actitud investigativa que indague por las necesidades e intereses de 
los sujetos, por los niveles de compromisos con la formación y estructuración intelectual, por los diferentes niveles de elaboración conceptual, por los contextos culturales que enmarcan a la comunidad educativa, por las distintas formas de relaciones que se manifiestan dentro del aula, etc., sólo de esta manera es posible desarrollar una práctica evaluativa que elimine las escalas de medidas y en cambio, atienda los diferentes ritmos de aprendizaje de cada uno de los estudiantes.

\section{Unas ideas finales}

Hemos manifestado que las prácticas evaluativas siempre se hacen de manera intencionada y que se hacen a partir de los modelos pedagógicos que nos instituye a cada uno. O sea, los imaginarios que poseemos acerca de dichas prácticas, determinan nuestras acciones en el ejercicio de evaluar. Por ello, no es gratuito que se evalúe para promover o para nivelar a los estudiantes o

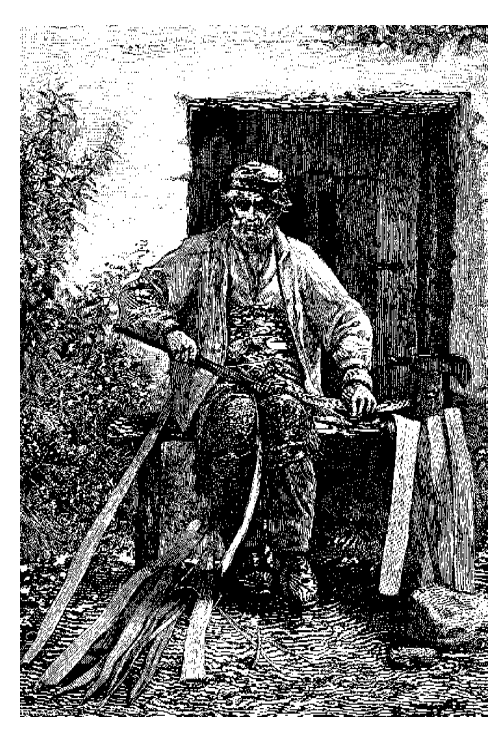

para medir conocimientos, etc. Ante esta circunstancia es necesario adoptar una actitud critica que cuestione permanentemente el trabajo que hacemos en la escuela, para generar discusiones con nuestra comunidad educativa con la finalidad de hacer otras miradas hacia la evaluación, buscando promover una evaluación de corte cualitativo que durante su práctica, genere conocimientos que propendan por la formación integral, no solo de estudiantes, sino también de maestros y padres de familia. Se trata de involucrar a los distintos actores que participan del acto educativo en la construcción colectiva de criterios que posibiliten el desarrollo de unas prácticas evaluativas que reconozcan los diferentes ritmos de aprendizaje, las individualidades de maestros y estudiantes, la integralidad, pero ante todo, que procure la formación de cada una de las personas que hacen presencia en la escuela.

Pero establecer una evaluación en nuestra institución con éstas características, exige posicionarse en unos referentes teóricos que den cuenta de procesos de problematización que promuevan una ruptura con las prácticas evaluativas que se vienen realizando a partir de los elementos propios de la evaluación cuantitativa. Esto es, se requiere instalar la evaluación como un proceso que genera conocimientos en donde las prácticas reclaman continuamente la construcción de sentidos y en donde la reflexión cobra un lugar privilegiado.

No es fácil asumir estas posturas, necesitamos seguir indagan- do y seguir interactuando con los miembros de nuestra comunidad educativa para desentrañar el entramado social que sirve de marco a nuestras acciones. El proceso investigativo en el que estamos empeñados tendrá que dar cuenta de posibles alternativas y de posibles respuestas a los interrogantes formulados anteriormente, que nos permitan posicionarnos de referentes teóricos y metodológicos para abordar nuestra práctica pedagógica y evaluativa, por lo pronto hasta aquí va el debate.

\section{BIBLIOGRAFÍA}

Caicedo, Lilián. 1998. "Vigilia evaluativa". En: Revista Contextos Educativos. No.1. Santa Fe de Bogotá.

JuRAdo, Fabio. 1998. Investigación, Escritura y Educación. Santa Fe de Bogotá: Universidad Nacional de Colombia.

JuRADO, Fabio y otros. 1998. Juguemos a interpretar. Santa Fe de Bogotá: Plaza y Janés.

MEN, 1994. Ley general de educación (Ley 115). Santa Fe de Bogotá: MEN.

1994. Decreto 1860. Santa Fe de Bogotá: MEN.

1997. La evaluación en el aula y más allá de ella. Santa Fe de Bogotá: MEN.

Pérez, Mauricio y Bustamante, G. 1996. Evaluación escolar. ¿Resultados o procesos? Santa Fe de Bogotá. Cooperativa Editorial Magisterio. 